Résumés des conférences et travaux

\title{
Initiation à la civilisation chinoise ancienne
}

Conférences de l'année 2014-2015

\section{Olivier Venture}

\section{(2) OpenEdition \\ Journals}

\section{Édition électronique}

URL : https://journals.openedition.org/ashp/1881

DOI : 10.4000/ashp.1881

ISSN : $1969-6310$

\section{Éditeur}

Publications de l'École Pratique des Hautes Études

\section{Édition imprimée}

Date de publication : 1 septembre 2016

Pagination : 310-316

ISSN : 0766-0677

Référence électronique

Olivier Venture, "Initiation à la civilisation chinoise ancienne ", Annuaire de l'École pratique des hautes études (EPHE), Section des sciences historiques et philologiques [En ligne], 147 | 2016, mis en ligne le 05 octobre 2016, consulté le 06 juillet 2021. URL : http://journals.openedition.org/ashp/1881 ; DOI : https://doi.org/10.4000/ashp.1881 


\title{
INITIATION À LA CIVILISATION CHINOISE ANCIENNE
}

\author{
Maître de conférences : M. Olivier VENTURE
}

Programme de l'année 2014-2015 : I. Actualité des découvertes et publications d'inscriptions, de documents et livres manuscrits de la Chine ancienne. - II. Particularismes régionaux dans les inscriptions sur bronze des Shang et des Zhou (env. XIII ${ }^{e}-I I I^{e}$ s. av. J.-C.) : le cas du Henan; partie 4 : Pingdingshan (suite et fin). - III. Lecture d'inscriptions chinoises antiques : inscriptions sur bronze de la cache 2003MYJ1 de Yangjiacun (VIII av. J.-C.).

I. Comme chaque année depuis 2009, le maître de conférences a consacré plusieurs séances à rendre compte des nouvelles découvertes et publications de matériaux épigraphiques, ainsi que de documents et livres manuscrits produits entre le milieu du $\mathrm{XIII}^{\mathrm{e}}$ siècle avant notre ère et la fin $\mathrm{du}_{\mathrm{III}}{ }^{\mathrm{e}}$ siècle de notre ère. L'année universitaire 2013-2014 a été en particulier marquée par la parution de plusieurs articles concernant la deuxième campagne du fouilles du site de Yejiashan 葉家山, près de Suizhou, au Hubei, qui est daté de la phase initiale des Zhou occidentaux (env. 1050-950 av. J.-C. ; voir revue Jianghan kaogu, 2013, nos 3 et 4, et 2014, n ${ }^{\circ}$ 1). Comme l'ont confirmé les inscriptions sur bronze découvertes lors de cette nouvelle campagne, plusieurs seigneurs de la principauté de Zeng 曾 étaient inhumés dans ce cimetière. Les débats entre spécialistes pour identifier les propriétaires des tombes à partir des inscriptions illustrent assez bien la complexité des relations entre inscriptions, mobilier funéraire et défunts. Ces découvertes viennent également enrichir le dossier épigraphique qui s'est constitué au sujet de cette principauté depuis la découverte de la tombe du marquis Yi de Zeng en 1977. Parmi les publications les plus importantes de manuscrits et de documents, on peut relever le volume 3 des documents administratifs et militaires découverts en 1973 sur le site de Jinguan 金關, au Gansu, dans la zone du limes occidental de l'empire Han (voir Jianshui Jinguan Han jian 肩水金關漢簡, vol. 3, Shanghai, Zhongxi shuju, 2013). Ces documents datent pour la plupart du premier siècle avant notre ère et début du premier siècle de notre ère. On doit également mentionner le volume 4 des manuscrits de l'université de Tsinghua, provenant du marché des antiquités et conservés depuis 2008 par cet établissement. Trois manuscrits de ce corpus, datant vraisemblablement des alentours de 300 avant notre ère sont présentés dans ce volume : le Shifa 筮法, manuel de divination rassemblant différentes méthodes pouvant être rattachées à la tradition du Livre des mutations ou Zhouyi 周易, le Biegua 別卦, courte liste de trigrammes, avec leurs noms, relevant de cette même tradition, et enfin le Suanbiao 算表, une table de multiplication concernant les nombres entiers de 1 à 9 , les dizaines 10 à 90 , ainsi que les demis et les quarts.

II. L'essentiel des conférences du premier semestre a été consacré aux inscriptions sur bronze provenant du cimetière aristocratique de Ying 應 à Pingdingshan 平頂山 (Henan). Notre analyse s'est principalement appuyée sur le premier volume du rapport 
de fouilles de ce cimetière : Pingdingshan Ying guo mudi 平頂山應國墓地 (Zhengzhou, Daxiang chubanshe, 2012). L'année dernière, nous avions traité l'ensemble des six tombes datées dans cet ouvrage de la phase initiale des Zhou occidentaux, ainsi que deux tombes de la phase moyenne (env. 950-850 av. J.-C.). Ce semestre, notre étude a d'abord porté sur les dix autres tombes datées de la phase moyenne des Zhou occidentaux. Seules deux tombes de ce groupe contenaient des vases inscrits. La tombe M210 renfermait cinq vases de qualité assez médiocre, presque tous dépourvus de décor, et un vase de meilleure facture, avec un décor d'oiseau assez élaboré. Sur ce dernier figurait une courte dédicace, ne mentionnant pas le nom de Ying. Dix vases en bronze avaient été déposées dans la tombe M84, dont sept comportaient une inscription. Dans trois cas sur sept, le dédicataire peut être identifié avec certitude comme un seigneur du pays de Ying (應侯). Pour les quatre autres inscriptions, l'identité exacte de la personne ayant fait fondre ces bronzes est plus incertaine. Les volumes suivants du rapport de fouilles se rapportant aux périodes postérieures n'ayant toujours pas été publiés, il ne nous a pas été possible de traiter de manière aussi exhaustive l'ensemble des autres tombes du cimetière de Pingdingshan. Pour poursuivre notre enquête, nous avons dû nous contenter d'une série de rapports de fouilles préliminaires, beaucoup plus partiels, publiés dans différentes revues archéologiques chinoises. Ainsi avonsnous étudié les tombes M95, M1, M8, M301 et M327, qui sont datées entre le milieu du VIII ${ }^{\mathrm{e}}$ siècle et le $\mathrm{IV}^{\mathrm{e}}$ siècle avant notre ère. Les informations données dans ces publications ont pu être complétées par des notes personnelles et des photographies prises, par le maître de conférences, dans le Musée de la province du Henan, à Zhengzhou, et dans le Musée de Pingdinshan, où sont conservées une partie des pièces archéologiques découvertes sur le site en question. Nous nous sommes également intéressés aux autres tombes Zhou de la région, ainsi qu'à des bronzes comportant des inscriptions mentionnant des membres du lignage Ying exhumés dans d'autres régions. Parmi les pièces n'ayant pas été découvertes lors de fouilles archéologiques, une attention particulière a été accordée à un ensemble de bronzes exhumés du cimetière de Ying à Pingdingshan par des pillards qui a pu être récupéré par la police. Le rapport de fouilles présente de nombreuses autres pièces, de provenance inconnue, dont les inscriptions mentionnent le lignage de Ying et que les auteurs associent à plusieurs tombes du site ayant été pillées entre l'époque des Song (960-1279) et ces dernières années. Mais il est difficile d'accepter l'ensemble des associations proposées dans cet ouvrage. D'abord, parce qu'on peut encore s'interroger sur l'authenticité de certaines pièces (comme le fait Lothar von Falkenhausen dans son article : « Bronzes of Ying and their Inscriptions », dans Zhongguo gudai qingtongqi guoji yantaohui lunwenji 中國古代 青銅器國際研討會論文集, Shanghai-Hong Kong, Shanghai bowuguan-Xianggang zhongwen daxue wenwuguan, 2010, p. 89-164) et ensuite parce que, comme l'attestent les découvertes archéologiques, tous les bronzes inscrits faisant référence à Ying ne viennent pas nécessairement du cimetière de Pingdingshan.

La plupart des tombes du site ayant été pillées, une grande prudence s'impose quant aux conclusions pouvant être tirées de l'analyse des vestiges encore préservés. Un certain nombre de remarques peuvent néanmoins être formulées. La taille et la structure des sépultures indiquent que le cimetière de Pingdingshan était bien un cimetière aristocratique, occupé depuis le début du $\mathrm{X}^{\mathrm{e}}$ siècle jusqu'au $\mathrm{IV}^{\mathrm{e}}$ siècle avant notre ère environ. La tombe M232, qui est une des plus anciennes du site et qui avait été 
entièrement pillée, partage de nombreuses caractéristiques avec des tombes de seigneurs locaux (zhuhou 諸侯), mises au jour dans d'autres régions. Cependant, celleci apparaît ici comme une exception, les autres sépultures du site étant beaucoup plus modestes. Les vases rituels en bronze étaient ici fréquents dans les tombes de la phase initiale des Zhou occidentaux et plutôt concentrés dans les sépultures les plus grandes à partir de la phase moyenne. On a aussi relevé que de nombreux vases étaient de qualité moyenne, voire très médiocre, les auteurs du rapport les considérant alors généralement comme des substituts à usage funéraire. Les quelques tombes postérieures pour lesquelles on dispose d'informations semblent s'inscrire cette tendance, observable sur la plupart des sites contemporains, qui se traduit par une baisse très significative, avec le temps, du nombre de vases en bronze inhumés, la tombe la plus tardive ne contenant plus, ici, que des copies en terre cuite de ces vases. Le fait que les substituts de vases rituels à usage funéraire ne comportent presque jamais d'inscription explique en partie que le fait que ces témoignages écrits semblent disparaître des tombes de Pingdingshan dès la phase finale des Printemps et automnes (env. 550-481 av. n. è.). Pour les périodes antérieures en revanche, des inscriptions sont présentes dans la très grande majorité des tombes contenant des vases rituels en bronze (en mettant de côté les sépultures les plus gravement pillées, qui ne contenaient plus que des fragments de vases, la proportion s'élève à sept tombes sur huit). En tout, dix-neuf vases en bronze inscrits ont été exhumés scientifiquement des tombes du cimetière de Pingdingshan datant des phases initiale et moyenne des Zhou occidentaux. À ceux-ci ont doit ajouter dix-sept vases inscrits provenant de tombes de la phase finale des Zhou occidentaux (850-771) et de la phase initiale des Printemps et automne (env. 770-650 av. n. è.). Ying correspond au nom le plus fréquent dans ces inscriptions. Il est vraisemblable que de nombreux membres de ce lignage étaient enterrés dans ce cimetière. Textes transmis et inscriptions s'accordent sur le fait que cette famille était à la tête d'un fief dans la région de Pingdingshan. Cependant, Ying n'est présent que dans treize des trente-six inscriptions étudiées et ces dernières ne proviennent en fait que de quatre sépultures (M229, M84, M95 et M8). Dans les autres inscriptions, différents noms apparaissent. La présence importante, dans ce cimetière, de vases fondus par des personnes ne se présentant pas explicitement comme des membres du lignage Ying pose problème. On a de relevé plusieurs exemples de tombes contenant des ensembles de bronzes anépigraphes, tout à fait ordinaires, au milieu desquels se trouvait un bronze inscrit d'une qualité tout à fait remarquable. Si le cimetière en question était bien essentiellement occupé par des membres du lignage Ying, on ne peut pas dire qu'il s'agissait là d'un lignage particulièrement prospère. En revanche, ce lignage entretenait manifestement des liens étroits avec le domaine royal des Zhou. Plusieurs textes anciens identifient le premier seigneur de Ying comme un membre de la famille royale. Il est également vraisemblable que certains membres de ce lignage aient occupé des positions élevées à la cour des Zhou. Dans ce contexte, la richesse exceptionnelle de certaines tombes du cimetière de Pingdingshan pourrait être liée, non à la prospérité du lignage, mais au statut particulier de quelques individus. Ainsi, en dehors de quelques défunts remarquables, la plupart des aristocrates enterrés à Pingdingshan disposaient d'un mobilier relativement modeste, dans lequel pouvait éventuellement se trouver un ou deux vases inscrits d'une qualité supérieure, pas nécessairement fondus pour le propriétaire de la tombe et acquis dans des circonstances très difficile à déterminer. 
En commençant ce travail de recherche, en 2008, à l'occasion d'une délégation au centre de Pékin de l'École française d'Extrême-Orient, je souhaitais avant tout m'intéresser aux spécificités régionales des inscriptions sur bronze des Shang et des Zhou. Pour cela, j'avais choisi de me concentrer sur la province du Henan et plus particulièrement sur quatre sites archéologiques. Mes analyses et conclusions ont été résumées dans les annuaires de 1'EPHE : Anyang (2009-2010), Luoyang (2010-2011 et 20112012), Sanmenxia (2012-2013) et Pingdingshan (2013-2014 et 2014-2015). Au bout de sept années de réflexion, je ne suis pas vraiment parvenu à mettre en évidence les spécificités régionales des inscriptions sur bronze. Je vois au moins deux raisons à cela. Il y a sans doute d'abord une uniformité des inscriptions, au moins jusqu'au vi ${ }^{\mathrm{e}}$ siècle avant notre ère, encore plus grande que ce que j'avais imaginé. La mobilité des objets inscrits constitue une autre difficulté. L'archéologie nous permet de connaître avec précision le lieu où l'objet a finalement été enterré, mais ce lieu n'est pas nécessairement celui où il a été produit. Dans ma thèse de doctorat (Étude d'un emploi rituel de l'écrit dans la Chine archaïque [XIII ${ }^{e}-V I I I^{e}$ s. av. notre ère] - réflexion sur les matériaux épigraphiques des Shang et des Zhou occidentaux, université Paris-Diderot, 2002), je m'étais interrogé sur les motivations de la production d'inscriptions dans la Chine antique. Le contexte dans lequel la plupart des inscriptions sur bronze étaient réalisées était le culte des ancêtres. Mais ici, c'est dans des sépultures que la plupart des vases inscrits ont été découverts et la question se pose de leur usage en contexte funéraire. La mise en évidence des relations entre bronzes inscrits et bronzes anépigraphes dans chaque tombe, au sein de chaque cimetière ou pour une époque donnée m'a permis de mettre en lumière différents phénomènes. En dehors des informations irremplaçables que peuvent fournir les inscriptions par leur contenu, on peut ajouter que leur forme, leur qualité, leur présence ou leur absence peuvent également apporter des renseignements précieux sur le statut d'un défunt en particulier ou sur les groupes d'individus ayant été enterrés au sein d'un même cimetière. La prise en compte de ces différents éléments oblige à sortir des frontières de l'épigraphie classique : à accorder plus d'importance aux relations entre l'inscription et l'objet qui lui sert de support, aux relations entre objets inscrits et non inscrits, aux usages de ces objets inscrits par les personnes de l'époque, etc. Ainsi, à côté de la nécessaire approche philologique des inscriptions, une approche plus archéologique me semble présenter un réel intérêt.

III. En 2003, des archéologues ont mis au jour à Yangjiacun 楊家村 (Meixian 眉 縣, Shaanxi) une cache datée du VIII ${ }^{\mathrm{e}}$ siècle avant notre ère qui contenait vingt-sept vases en bronze. Cet ensemble se distingue d'une part par la qualité remarquable de la plupart des pièces et d'autre part par le fait que celles-ci présentaient toutes une inscription. On compte en outre parmi ces inscriptions des documents tout à fait exceptionnels.

Plusieurs séances ont d'abord été consacrées au contexte de cette découverte d'origine accidentelle et aux conditions difficiles dans lesquelles la fouille a été menée. On s'est ensuite interrogé sur la cache elle-même, dont la forme ne correspond pas aux standards de cette époque. L'hypothèse la plus vraisemblable est que, dans un premier temps, une tombe aurait été creusée, mais pour des raisons qui nous échappent, celleci n'aurait pas été utilisée comme telle et c'est finalement un ensemble de vases rituels 
en bronze que l'on aurait décidé d'enfouir dans cette fosse. On considère généralement que c'est parce qu'elles avaient décidé de fuir la région face aux avancées d'ennemis venant du nord que de nombreuses familles aristocratiques installées dans cette région décidèrent d'enterrer ces biens certes précieux, mais lourds (l'ensemble de la fosse de Yangjiacun pèse plus de $390 \mathrm{~kg}$ ), sans doute avec l'idée de revenir plus tard les chercher, une fois le danger passé.

Des comparaisons entre les vases de cette fosse et de nombreuses pièces contemporaines ont permis de mettre en évidence la qualité des pièces et leur taille remarquables. Il s'agit manifestement de bronzes fondus dans les meilleurs ateliers du domaine royal des Zhou, probablement des ateliers royaux. L'ensemble comprenait douze tripodes ding 鼎 et neuf vases $l i$ 鬲, en principe réservés à la cuisson des viandes, deux grandes jarres $h u$ 壳 pour contenir des boissons alcooliques, un bassin pan 盤 et deux verseuses he 盉 et $y i$ 巴 pour les ablutions, ainsi qu'un bassin yu 孟. L'absence de certaines formes de vases très importantes dans la vaisselle rituelle de l'époque, comme les vases à céréales gui 笽 et $x u$ 須, indique que l'ensemble en question ne correspond probablement qu'à une partie des vases rituels qui devaient à l'origine se trouver dans le temple ancestral de cette famille. Il s'agit donc de pièces qui ont été choisies avant d'être placées dans cette cache.

Deux grands vases ding portaient une longue inscription au contenu pratiquement identique. Le texte commémore une cérémonie de don ayant eu lieu le cinquième mois de la quarante-deuxième année du règne d'un roi dont le nom n'est pas précisé. Sur les dix autres vases ding, de tailles décroissantes, figurait une autre longue inscription au sujet d'une cérémonie d'investiture au profit du même personnage que dans l'inscription précédente. La cérémonie est ici datée du sixième mois de la quarante-troisième année. La plupart des chercheurs considèrent que ces deux groupes de vases ont été fondus sous le règne du roi Xuan 宣 des Zhou (env. 827-782). Les deux inscriptions posent encore de nombreux problèmes de compréhension, mais en nous basant sur les nombreuses études publiées principalement en langue chinoise, et à la suite des professeurs Shaughnessy, Falkenhausen et Li Feng, nous avons proposé notre propre traduction de ces deux textes. Pour cet exercice difficile, nous avons souhaité signaler par des « $[\mathrm{X}] »$ les passages qui restent, selon nous, incompréhensibles et par l'usage de l'italique associé à des points d'interrogation entre parenthèses des lectures possibles, mais qui attendent encore d'être confirmées.

Inscription du vase ding de Lai de la quarante-deuxième année (四十二年逨鼎) :

Lors de la $42^{\mathrm{e}}$ année, au $5^{\mathrm{e}}$ mois, après la « clarté naissante », au jour yimao (jour 52/60), le roi était dans le temple du (roi) Mu (du temple) du (roi) Kang à Zhou. À l'aurore, le roi se rendit dans la Grande salle et y prit place. Le Directeur des travaux San aida le Responsable des eaux et des forêts Lai à franchir la porte et à se tenir dans la cour centrale, en faisant face au nord. Le Chef d'office donna au roi le document de gratification. Le roi appela le Scribe Huo pour qu'il procède à la gratification de Lai conformément au document officiel (?). Le roi s'exprima ainsi : «Lai ! Les très illustres (rois) Wen et Wu avaient accepté de recevoir le Grand mandat et l'avaient étendu aux quatre orients, dès lors (?) vos sages aïeux et père défunts qui vous ont précédé ont assisté les anciens rois, ils se sont donné de la peine (?) pour le Grand mandat et ont stabilisé le domaine des Zhou. Je ne peux (?) pas oublier les descendants des sages. J'ai bien connu (?) vos 
aïeux et père défunts qui vous ont précédé et qui se sont dépensés (?) pour le domaine des Zhou. C'est pourquoi, j'ai fait [X X X]. J'ai pour la première fois établi Changfu en tant que seigneur à Yang. Je vous ai ordonné de stabiliser le bien de Changfu. Vous avez pu apporter de la stabilité à ses troupes. Vous avez vraiment été capable de prendre modèle sur vos aïeux et père défunts qui vous ont précédé. (Vous avez) $[\mathrm{X}]$ le soulèvement des Xianyun et les avez vaincus à Jing'a et à Liyan. Vous n'avez pas [X] les Rong. Vous avez [X] Changfu en poursuivant les Rong pour les combattre. Une fois rattrapés, vous avez fondu sur eux (?) à Gonggu. Vous avez capturé des prisonniers, vous avez obtenu des têtes coupées, vous vous êtes emparé de leur matériel, de leurs chars et de leurs chevaux. Vous vous êtes montré appliqué dans les travaux militaires. Vous ne vous êtes pas opposé à l'ordre que je vous ai donné en personne. Je vous gratifie d'un you d'alcool de millet noir parfumé, de trente tian de terres cultivables à Zhi (?) et de vingt tian de terres cultivables à $Y i($ ?) ». Lai salua avec les mains et se prosterna en touchant le sol avec son front. Il reçut un document officiel et ses gratifications et sortit avec. (Moi) Lai j'ose, en réponse aux très illustres et excellents bienfaits du Fils du Ciel, lui présenter mes louanges. En cette occasion, j'ai fait ce vase rituel pour les sacrifices. Qu'il soit utilisé pour présenter des offrandes et manifester ma piété filiale à mes distingués prédécesseurs. Qu'ils soient majestueux tout là-haut et qu'ils soient respectés icibas. Qu'avec dignité, ils gardent leur brillante vertu, et que, plein d'ardeur et de vigueur (?), ils fassent descendre sur moi une paisible joie (?) et une pure assistance, une félicité pénétrante et un mandat éternel, une grande longévité, large et ample, et (que je puisse) avec excellence servir le Fils du Ciel. Que (pour moi) Lai, pendant dix-mille années, sans limite, les fils de mes fils et les petits-fils de mes petits-fils le conservent précieusement et éternellement et l'utilisent pour faire des offrandes.

Inscription du vase ding de Lai de la quarante-troisième année (四十三年逨鼎) :

Lors de la $43^{\mathrm{e}}$ année, au $6^{\mathrm{e}}$ mois, après la «clarté naissante », au jour dinghai (jour $24 / 60$ ). Le roi était dans le temple du (roi) Mu du temple du (roi) Kang à Zhou. À l'aurore, le roi se rendit dans le temple ancestral des Zhou et y prit place. Le Directeur des chevaux Shou aida le Responsable des eaux et des forêts Lai à franchir la porte et à se tenir dans la cour centrale, en faisant face au nord. Le Scribe Huo donna au roi le document d'investiture. Le roi appela le chef d'office, pour qu'il procède à l'investiture de Lai conformément au document officiel (?). Le roi s'exprima ainsi : « Lai ! Les très illustres (rois) Wen et Wu avaient accepté de recevoir le Grand mandat et l'avaient étendu aux quatre orients, dès lors (?) vos sages aïeux et père défunts qui vous ont précédé ont assisté les anciens rois, ils se sont donné de la peine (?) pour le Grand mandat et ont stabilisé le domaine des Zhou. Je n'oublie pas les descendants des sages. Je vous ai déjà autrefois ordonné d'assister Rong Dui, et d'administrer les responsables des eaux et forêts des quatre orients, pour le service du Palais. Aujourd'hui, prenant en considération le fait que (?) vos aïeux et père défunts qui vous ont précédé et se sont dépensés (?) pour le domaine des Zhou, j'augmente et complète votre charge. Je vous ordonne d'administrer les liren. Vous ne devrez pas oser vous montrer négligeant ou oisif. Respectueusement, jour et nuit, vous vous montrerez bienveillant et ferez régner la concorde entre les grands et les petits projets au sein de notre domaine. Dans votre charge de mise en application de la politique du gouvernement, vous n'oserez pas ne pas vous conformer aux standards (?) et aux règles. Quand vous interrogerez les shu, vous vous montrerez perspicace (?), vous n'oserez pas ne pas être juste ou ne pas vous conformer aux règles. Vous ne devrez pas [XX], car [XX] conduit à l'indulgence, et cela reviendrait à traiter avec mépris les veufs et les veuves âgées. Ainsi, moi l'homme 
unique je [X], et [vous] mourrez (?) sans titre (?) ». Le roi dit : « Lai, je vous confère un you d'alcool de millet noir parfumé, la robe sombre, les chaussures rouges, une voiture à cheval, dotée d'un jiao décoré et d'une pièce recouvrant la barre d'appui, ainsi que d'un jin, tous les deux en cuir de couleur vermillon, un dais en peau de tigre avec une doublure rouge pâle, des $f u$ et des jantes (?) décorés, une cloche en bronze, (ainsi que) quatre chevaux et une bride avec des ornements en métal. Qu'avec respect, nuit et jour vous n'abandonniez pas le mandat que je vous ai donné ». Lai salua avec les mains et se prosterna en touchant le sol avec son front. Il reçut le document officiel et sortit après l'avoir suspendu à sa ceinture. Il revint et offrit un jade jin et un jade gui. (Moi), Lai, j'ose, en réponse aux très illustres et excellents bienfaits du Fils du Ciel, lui présenter mes louanges. En cette occasion j'ai fait, pour mon Auguste père défunt Gong Shu, ce vase rituel pour les sacrifices. Que mon Auguste père défunt soit majestueux tout là-haut et qu'il soit respecté ici-bas, qu'avec dignité, il garde sa brillante vertu, et que, plein d'ardeur et de vigueur (?), il fasse descendre sur moi une paisible joie (?) et une pure assistance, une félicité pénétrante et un mandat éternel, une grande longévité, large et ample, et (que je puisse) avec excellence servir le Fils du Ciel. Que (pour moi) Lai, pendant dix-mille années, sans limite, les fils de mes fils et les petits-fils de mes petits-fils, le conservent précieusement et éternellement et l'utilisent pour des faire des offrandes.

Une analyse de ces deux textes sera proposée l'année prochaine, après avoir mené à bien la lecture et la traduction d'une des plus longues inscriptions de l'époque des Zhou occidentaux, qui se trouvait également dans cette cache : celle du bassin pan de Lai (逨盤). 